\title{
Prototype of Parking Finder Application for Intelligent Parking System
}

\author{
Lana Benny", Prashant Kumar Soori" ${ }^{* *}$ \\ \# Department of Electrical and Electronics Engineering, Heriot-Watt University Dubai Campus, Dubai, U.A.E. \\ E-mail:lbt30@hw.ac.uk; *p.k.soori@hw.ac.uk
}

\begin{abstract}
This paper explains the design and fabrication of an intelligent, user-friendly parking system developed in the United Arab Emirates. The need for a smart parking system was assessed by conducting a survey on the current parking issues. This paper elaborates on the hardware and software realization of the proposed parking system developed for motorists to locate vacant parking using mobile application. The various types of vehicle detection sensors available in the market have been evaluated for the implementation of the system. The main objective of this paper is to build a prototype intelligent parking system with maximum accuracy. The goal of the project was to control the detection modules wirelessly through a customized mobile application, allowing ease of operation and maintenance. While the users can enjoy better comfort and safety, the focus is to create a self-reliable, and ecologically sustainable system while reducing searching time, fuel wastage resulting in lower carbon footprint. The mobile application has been developed using Android Studio, and the results are presented in this paper.
\end{abstract}

Keywords - mobile application; parking system; real-time data transfer; ultrasonic sensor

\section{INTRODUCTION}

Nowadays advancements to develop smart cities have created a shift to harness artificial intelligence for the growth of humanity. Across central districts and downtowns of populated cities, consumers and companies alike cite the lack of available parking as one of the major problems. The cause of this issue is due to the number of vehicles on the road exceeding the availability of parking space in highly populated areas. Hongwei Wang et al. came up with a reservation based system using light and vibration sensors [1]. However, it was reported that the effects of environment impairments due to false detection or the natural factors would affect the light sensors. Srikanth et al. implemented a Smart PARKing (SPARK) system using Wireless Sensor Network, but it seemed unfeasible as the selected sensor was weak against various environmental conditions [2].

Using a different technology, Gupta et al. proposed an intellectual context-aware parking space via computing, information stations, and swarm intelligence [3]. It detects parking spaces by mapping the shortest route to the base station with the help of the sensors. In a research by Mingkai Chen, a wireless sensor system which contains parking guidance and an information system is implemented. The system contains nodes for space monitoring, routing, guidance and information management [4]. The information is transmitted using wireless technology between the nodes where the protocol works using a tree-like structure. After analysing the collected data, the information is displayed on
LED screens. The results of the experiment showed that visual guidance is a more reliable option for drivers to locate parking.

Atif y. have used embedded wireless sensor networks to connect the status of parking space infrastructures and communication technology [5]. The model takes publically available data from the internet (such as Parkopedia www.parkopedia.se) and traffic counts (GPS, street cameras). The collected data is used to estimate the availability of realtime parking spaces with the help of probability. In addition, it automatically determines when and where drivers park their cars, and when parking spots are released.

Iris-net suggested using video cameras, motion detectors, and microphones. Since these devices are capable of detecting the availability of a parking lot, they were used [6]. The system procures real-time information regarding the parking spaces from web based applications. However, the data generated is quite large and results in high power consumption.

In the Telegraph UK, a survey conducted by 'Park at my House' in 2013 revealed that it takes an average of six minutes and forty-five seconds to find a suitable parking space [7]. The U.A.E. is working to make $25 \%$ of its transportation driverless by the year 2030 [8]. This strategy shall combat traffic congestions, road accidents, and pollution while saving millions of driving hours.

This paper discusses the implementation of a system which can tackle various parking related issues, by revealing parking availability to motorists. Providing an improved 
utilization of available parking spots, it creates a less congested, safer and pleasant environment. A system is designed, a prototype is built and tested successfully. The ultrasonic sensor in the ground unit of the proposed system can detect the presence of the vehicle irrespective of the type of vehicle to be parked. The module powers itself using solar energy and has a secondary battery for backup. The occupation status of the module is sent to a server using radio communication and can be modified to a mesh networking system for enhanced security. The server then sends this information to a database built in this prototype unit. The mobile application then uploads the data of the parking space to the user. The information in the database can also be used in future road planning and navigation. With the recent technological advancements in smartphone applications, such a system would be beneficial to drivers. The collected data could be linked to an Internet of Things (IoT) system which can be enabled to send information to predict the availability of parking spaces at a specified time of the day.

\section{MATERIAL AND METHOD}

\section{A. Survey Analysis}

A survey with a sample size of 100 people, was conducted to get a better understanding of the problems faced by residents. The results showed that the daily average time for parking ranged from one to fifteen minutes with $44 \%$ finding parking space within the first five minutes and $28 \%$ in six to fifteen minutes.

It was observed that majority of the participants (56\%) were from the age group of 18 to 24 years, followed by $22 \%$ from the age group of 45 to 54 years. There was a $12 \%$ response from the age group of 25 to 34 years and the least response, $2 \%$, were obtained from the 55 to 65 -year-oldcitizens. The majority of the respondents, $40 \%$, were from the emirate of Dubai, followed by residents of Abu Dhabi $(38 \%)$. There was a $17 \%$ response from Sharjah residents and $5 \%$ from the residents of the Northern Emirates (Al Ain, Ajman, and Ras Al Khaimah).

Over $92.93 \%$ responded 'Yes' to the need for a mobile application to locate available parking. It was concluded, that the rejection was because the respondents were from an emirate that faced minimal stress to locate parking. Thus, the need for an application to display parking availability in their environment was not a requirement.

Selection of preferred mobile application features was asked as one of the questions (Fig. 1), and it required participants to select options they would like to have on the application. Some additional suggestions from the consumers included using credit card payment for the parking fee, displaying the remaining time before additional payment and an option to view live traffic data for navigation and guidance.

Table 1 indicates that the average time taken to find parking during a weekday and weekend were largely similar. From the results, it was observed that $50 \%$ of the parking was located within the first 5 minutes. However, the remaining took more than five minutes to locate an available parking spot. The likely cause the respondents are able to park within the first five minutes is that they are given an allocated parking spot, thus, influencing the data collected.

What features would you look for in this

Application ? (Multiple Answers Permitted)

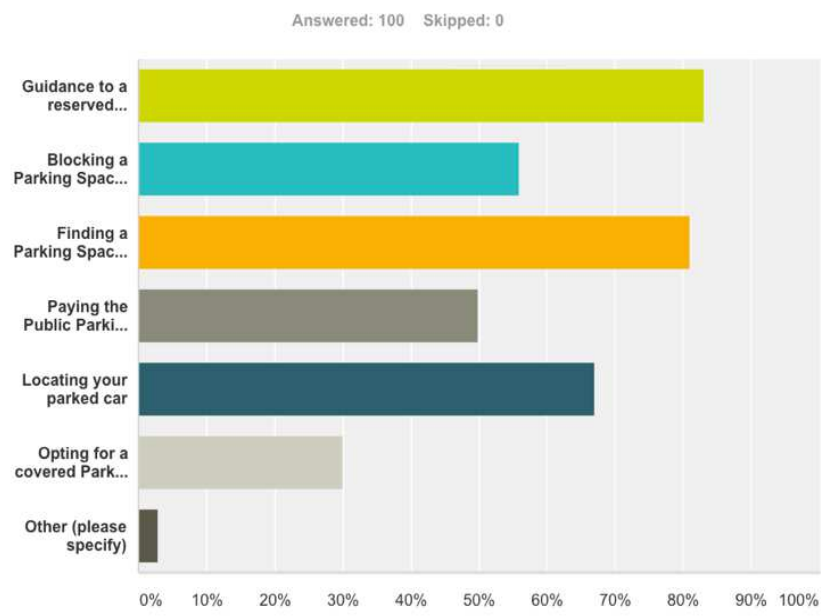

\begin{tabular}{|l|l}
\hline Answer Choices & Responses \\
\hline$\quad$ Guidance to a reserved parking & $83.00 \%$ \\
\hline $\begin{array}{l}\text { Blocking a Parking Space } 10 \text { minutes ahead of your arrival for a fee (includes } \\
\text { parking fee) }\end{array}$ & $56.00 \%$ \\
\hline$\quad$ Finding a Parking Space near you & $81.00 \%$ \\
\hline$\quad$ Paying the Public Parking Fee & $50.00 \%$ \\
\hline$\quad$ Locating your parked car & $67.00 \%$ \\
\hline Opting for a covered Parking Space with additional charges & $30.00 \%$ \\
\hline Other (please specify) & $30.00 \%$ \\
\hline
\end{tabular}

Fig. 1 Feature preference in mobile application

TABLE I

Average Time TAKEn to Find PARKING ON WEEKEND AND WeEKdAys

\begin{tabular}{|l|l|l|}
\hline \multirow{2}{*}{ Answer Choices } & \multicolumn{2}{c|}{ Responses } \\
\cline { 2 - 3 } & \multicolumn{1}{|c|}{ Weekday } & Weekend \\
\hline $0-5$ minutes & $52.53 \%$ & $44.90 \%$ \\
\hline $6-15$ minutes & $21.21 \%$ & $28.57 \%$ \\
\hline $16-30$ minutes & $19.19 \%$ & $17.35 \%$ \\
\hline 1 hour & $5.05 \%$ & $6.12 \%$ \\
\hline More than an hour & $2.02 \%$ & $3.06 \%$ \\
\hline
\end{tabular}

\section{B. Types of Sensors}

During the search for a viable sensor that can be used for vehicle detection, research was conducted to choose the best sensor suitable for the most efficient design.

1) Inductive Loops: Inductive loop detectors are commonly used for vehicle and speed detection. It is capable of estimating the length of the vehicle yet it is easily affected by temperature changes and traffic flow. The installation and maintenance of this sensor require lots of planning since it needs to be placed beneath the road surface.

2) Microwave Radar Detector: Microwave radar sensors are typically implemented to determine the speed and length for vehicle classification. They are insensitive to extreme weather but cannot detect stationary vehicles. 
3) Ultrasonic Detector: Ultrasonic sensors detect stationary vehicles to determine occupancy. They are one of the sturdiest sensors when it comes to varying climatic conditions. In addition to periodic maintenance checks, the sensors can be placed on the ground with ease.

4) Acoustic Sensor: Acoustic sensors are insensitive to moisture and light. They are perceptible to cold temperatures thus making it an unreliable installation option in open areas.

5) Magnetometer Sensor: The magnetometer sensors detect vehicle using magnetic signatures. They are less sensitive than inductive loops. It is best suited for vehicle and speed detection. The major challenge is the installation and maintenance of these sensors as they are typically placed underground. They also require customized algorithms to adapt to the location of installation.

6) Video Image Vehicle Detection Systems: It is commonly implemented to measure traffic flow, accident detection, lane occupancy and vehicle speed. The device has a high power consumption due to image detection and processing.

7) Weight Detection System: The weight detection system is mainly used to count and classify vehicles. There are few ways to implement such types of system. Road tubes are the most commonly used method. However, it is highly inaccurate in the presence of heavy vehicles. Pressure sensors for weight detection are reliable but tend to break easily and are susceptible to lightning.

8) Laser Scan: One of the most viable vehicle detection sensors, laser scan can be used for vehicle classification and weight detection. It requires no additional components for implementation thus making it expensive.

Wi-Fi and General Packet Radio Service (GPRS) with vehicle detectors are under development, but they are currently at a conceptual and testing phase.

Taking into account the merits and demerits of the various sensors explained above, ultrasonic sensors are used in the development of the proposed system. It is taken into consideration that non-invasive devices have the advantage of easier maintenance and installation. The Ultrasonic
Sensor is a distance sensor which sends out a high-frequency sound pulse. It then times the echo of the sound until it reflects back. It has two openings on the front. One transmits ultrasonic waves, (like a speaker), while the other receives them, (a microphone).

Since the speed of sound is approximately 341 meters per second $(\mathrm{m} / \mathrm{s})$ in the air, the ultrasonic sensor uses this information and the time difference to determine the distance between the sensor and the obstacle.

\section{Methodology}

The proposed system comprises of a field device, communication device and the front-end device as in Fig. 2. The system architecture for the prototype system was created using the application ' $\mathrm{X}$ - mind'. It is a project mapping software used to create block structures to highlight the various outcomes, issues, and paths that can be experienced by the system.

1) Field Device: An installation of the field device comprising of the vehicle detection unit is done in each parking lot. The module which consists of an ultrasonic sensor is used to identify the presence of a vehicle [9]. The microcontroller used for processing the information is an Arduino Uno [10]. The main feature of this device is the ability to detect modules with optimal accuracy. To power, the module continuously, harnessing solar energy to power the main battery is chosen. A backup battery is placed to prevent any possible power failure. A tri-colour Light Emitting Diode (LED) is placed on the device to help the motorists to view the availability status of the slot visually.

The Arduino code uses basic $\mathrm{C}$ programming to perform its functions. For the field device, the code is constructed such that when the sensor locates a vehicle between a distance of $0 \mathrm{~cm}$ to $50 \mathrm{~cm}$, the LED lights 'Red' indicating an occupied status. The code regularly checks for a change in distance in the background. The sensor has been programmed to check every $1500 \mathrm{~ms}$ so that there is no delay in updating the parking lot status in the database. The method is also implemented when the parking space is empty. When the sensor does not detect any object, the ultrasonic sensor lights the LED green (if there are no obstacles beyond $51 \mathrm{~cm}$ ) and value ' $\mathrm{V}$ ' is sent as output.

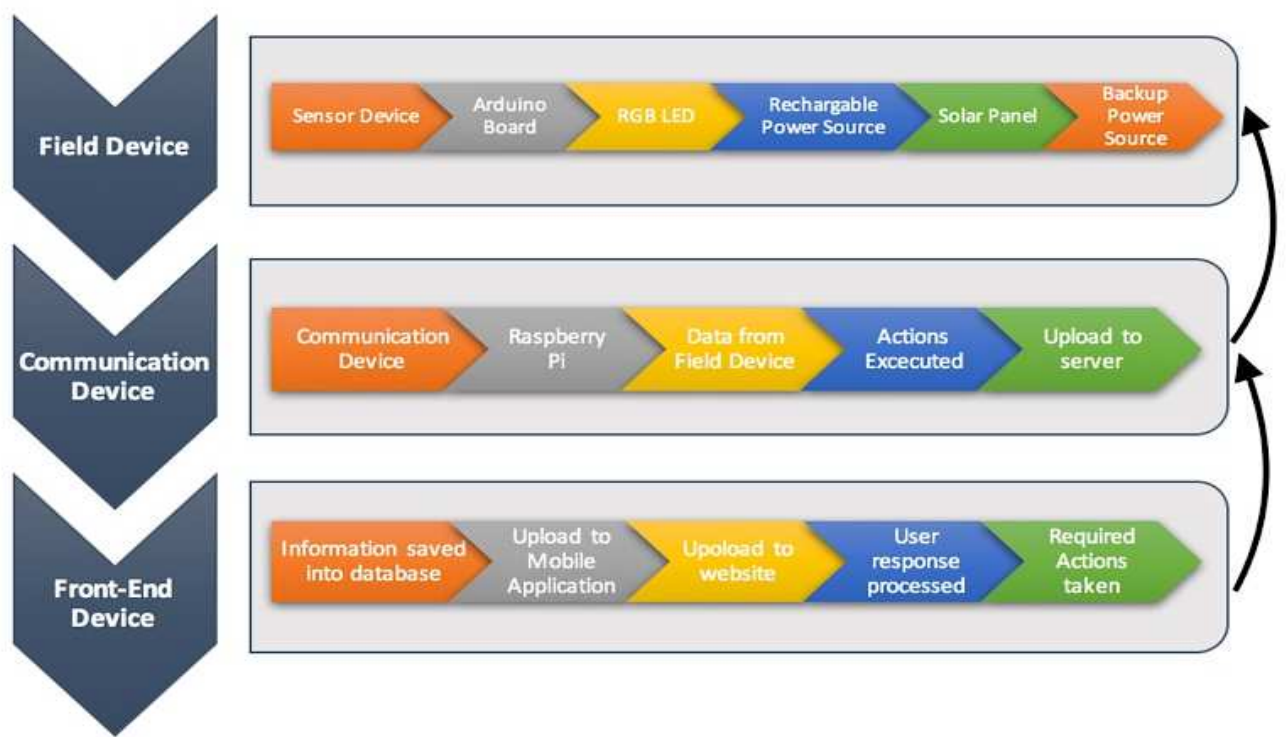

Fig. 2 Schematic of the system 
2) Data Communication: The sensor data from the field device is transferred with the help of a wireless transceiver (nRF24L01+) to a data collection hub. The nRF24L01+ is a single chip $2.4 \mathrm{GHz}$ transceiver, suitable for low power wireless functions [11]. It is designed for operation in the worldwide Industrial, Scientific, and Medical radio bands [ISM frequency band].

To design a radio system with the nRF24L01+ requires only a microcontroller and a few additional components to supply readings. Its internal voltage regulators ensure a high Power Supply Rejection Ratio (PSRR) and a wide power supply range. The Raspberry Pi microprocessor acts as the server and uploads this data to an online database called Firebase [12]. The addition of a suitable decoupling capacitor is required to avoid any data loss during communication. This is because an Arduino tends to supply less than $3.3 \mathrm{~V}$ when plugged into the $3.3 \mathrm{~V}$ pin. Due to an insufficient voltage supply, the data transferred gets affected. After testing various capacitors, a $4.7 \mu \mathrm{F}$ capacitor, proved to reduce the data loss.

On the Raspberry Pi, an Arduino Uno is connected using a serial connection. The microprocessor has an nRF24L01+ module connected to its pins making it the data collector for the server. This transceiver is programmed to collect the information from the field device on its assigned channel. The module after receiving the data from the field transceiver displays it on the Raspberry Pi terminal. A python code is used to collect the data from the Arduino serial monitor and then to Firebase.

To use Raspberry Pi as a web server, a LAMP (Linux, Apache, MySQL, PHP) stack must be set-up. The configuration to make the $\mathrm{Pi}$ work as a web server was completed and WordPress was downloaded and installed. A basic website is thus set up which can be accessed on the same network as the Raspberry Pi.

3) Mobile Application: The online database Firebase is a mobile and web application platform with flexible tools to help developers build high-quality apps [13]. It consists various features that developers can implement to fit their needs. The data from Firebase is collected in real-time and can be seen by motorists via a mobile application.

An application was constructed using Java on Android Studio (Fig. 3). The data from Firebase is collected by the mobile application 'Parking Finder' when it is connected to the Internet. A new user will be prompted to sign-up using necessary details, while the registered users will have access to multiple features. A few available options in the mobile application are paying the parking fee, recollecting vehicle location and guidance to a vacant space in their vicinity. The chosen option by the user would be implemented by sending commands back to the Hub and in turn the field device.

Java programming was executed to run the application on a Samsung S4. While a Login page and Registration page required minimal build. The creation of the core page to display the parking status required extensive research and code editing.

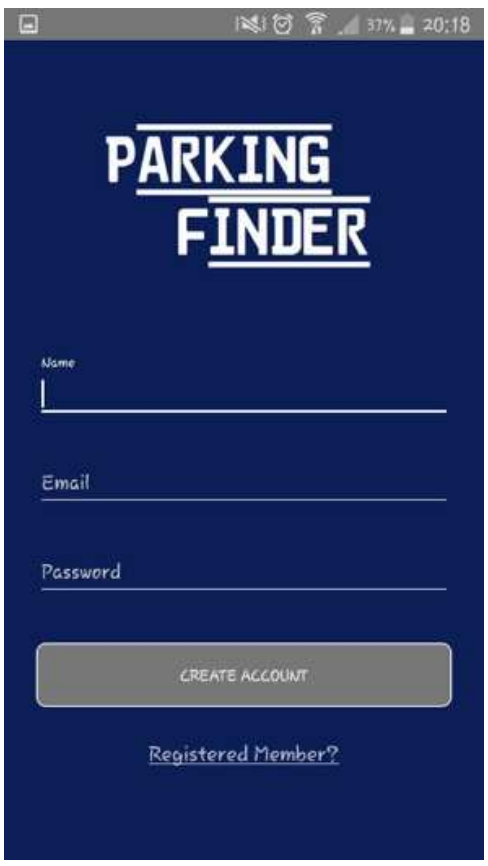

Fig. 3 Home page of mobile application

\section{Module Design}

While considering the hardware for the ground unit, it was important to restrict the power consumption low thereby extending the life of the solar charged batteries as well as the backup battery. Due to the availability of solar energy, an Arduino Uno was selected as the microcontroller for ease of prototyping. To ensure the safety of the components inside the module, a hard casing was built to accommodate the requirements. A viable prototype design was implemented using 3-D printed casings. It proved to be an efficient way of reducing the effects of the crash from the vehicles and helping protect the components housed on the ground. The placement of the ultrasonic sensors and the remaining components of the field device was decided after performing various test runs, analysis, and calculations. This was essential as even a slight error would result in an inaccurate reading leading to system errors. The raw data indicating the distance of the vehicle from the ultrasonic sensor was obtained using an Arduino code. A pulse from the sensor is sent from the trigger pin for a duration of 10 microseconds. The distance of the obstacle is calculated from the duration function which converts the time to into distance using equation (1).

$$
\text { Distance }=\frac{\text { Time } \times \text { Speed of sound }}{2}
$$

Time is measured in seconds.

Speed of sound is taken as $341 \mathrm{~m} / \mathrm{s}$

It is divided by two because the sound wave has to travel to the object and back after striking an obstacle.

After deciding on the proper combination of the battery, sensors and communication module the mobile application was built using Android Studio. A basic framework of the application was completed and tested with the help of the firebase data for a real-time transfer test. 


\section{RESULTS AND DISCUSSION}

\section{A. Sizing of power supply units}

The field device includes three components controlled by a microcontroller. Two different options considered are Arduino UNO microcontroller and Arduino pro-mini. For supplying power to the module, the battery is used. The sizing of the battery is calculated for both the microcontrollers and presented below.

- Case 1: Using an Arduino Uno

Components used: Ultrasonic sensor, NRF24L01+ module, and tricolour LED

Total power consumption $=$ Sum of power consumed by each module $=0.844 \mathrm{~W}$

Worst case scenario - Module is ' $O N$ ' for 24 hours Energy consumption, $\mathrm{E}=20.25 \mathrm{Wh}$

For the sizing of solar energy, the amount of sunlight the region receives must be considered. According to the collected data, the optimum sunlight hours is 6 hours a day [14].

Realistically, while doing solar sizing, a minimum of three days must be considered as the number of autonomous days. Therefore, the solar panel rating of $10.125 \mathrm{~W}$ is needed. The 11W solar panel from Gallagher is chosen [15].

Battery capacity calculations for one autonomous day: To select an optimum battery to meet the system requirements, the current and voltage ratings must be estimated.

Ampere hour rating $=2.64 \mathrm{Ah}$

Voltage Ampere rating $=1.32 \mathrm{~V}$

\section{- Case 2: Using an Arduino Pro Mini}

Components used: Ultrasonic sensors, NRF24L01+ and tricolour LED

Total power consumption $=0.34 \mathrm{~W}$

Worst case scenario - Module is 'ON' for 24 hours Energy consumption, $\mathrm{E}=8.16 \mathrm{Wh}$

If the region receives 6 hours of sunlight a day, the realistic solar panel rating with days of autonomy would be 4.1 W.

The current and voltage ratings estimated for the battery with one autonomous day;

Ampere hour rating $=1.0625 \mathrm{Ah}$

Voltage Ampere rating $=0.531 \mathrm{VA}$

Thus it can be concluded that using an Arduino Pro Mini or a similar chip is the viable option for the field device installation due to its smaller size and lower power consumption. However, Arduino Uno is selected for the design and development of this system.

\section{B. Results}

A prototype was built for the vehicle detection module using an ultrasonic sensor, and the same is depicted in Fig. 4. The module was tested with different types of vehicles (Fig. 5 ). The output data would be ' $\mathrm{V}$ ' for a vacant parking space which would turn the LED light to green. It sends the message ' $\mathrm{O}$ ' to the database for an occupied parking lot while turning the LED light to red.

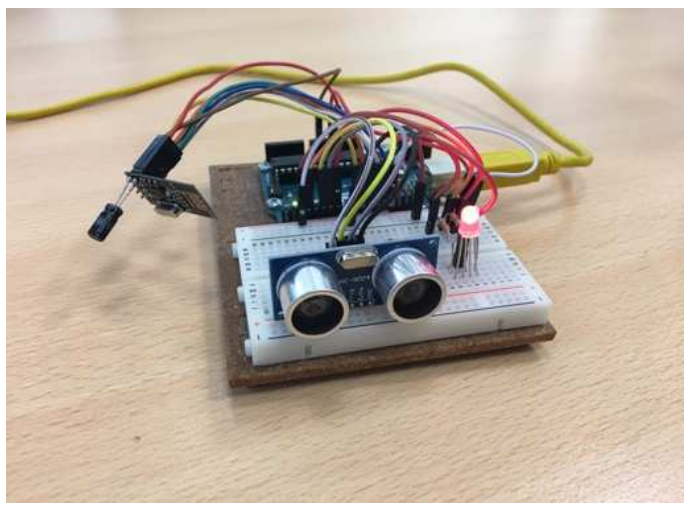

Fig. 4 Field device module

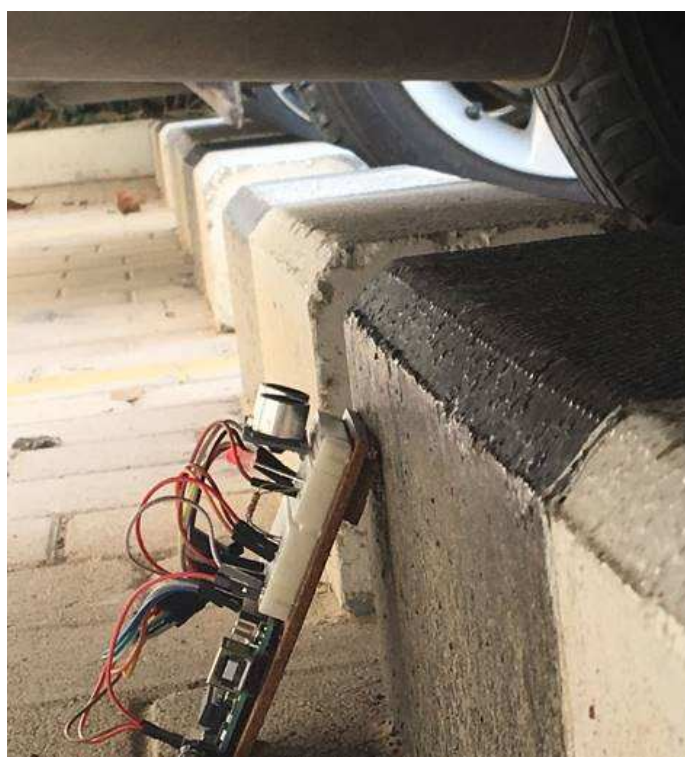

Fig. 5 Field testing

While the program was simulated, dips in the serial plotter were spotted. The dips are due to minor faults located in the sensor from manufacturing. This problem was overcome by replacing the existing sensor with a new ultrasonic sensor.

Fig. 6 shows the results obtained from the field device on the online database. The Raspberry $\mathrm{Pi}$ server code is programmed to send the status information to Firebase only when there is a change from the previous sensor reading. This reduces the energy consumption on the server. A delay in uploading the status information to Firebase is likely due to the speed of the network connected. 


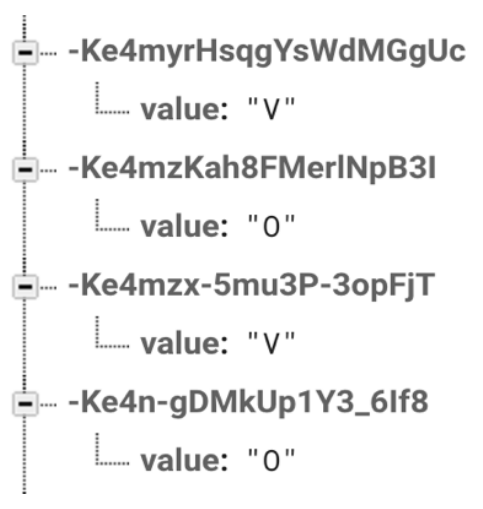

Fig. 6 Field device data on Firebase

The saved data on Firebase is acquired by the mobile application created on Android Studio. The application is called 'Parking Finder' and according to the results received from Firebase the status is displayed (Fig. 7) on the users mobile for viewing.
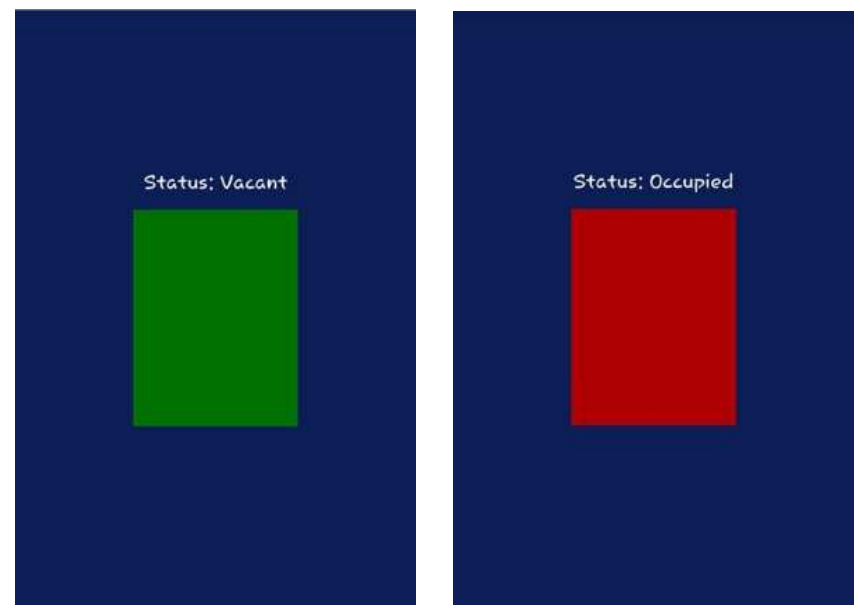

Fig. 7 Results observed on Mobile Application 'Parking Finder'

After multiple field tests and analysis, it was concluded that the system works quite robustly as no major flaws were detected. With periodic maintenance checks, the system can work at its maximum capability.

An option of using thermoelectric charging system or a substitute method could prove more viable. More testing and research need to be done to find an alternate battery charging source.

\section{CONCLUSIONS}

The prototype system was designed, built and tested successfully. The proposed system shall avoid traffic congestion caused while commuters search or wait for vacant parking spots. It presents ways to reduce the emotional and economic toll faced by the drivers and to alleviate the setbacks faced due to insufficient parking spaces. A way to improve communication between the mobile application and the field device must be done by implementing a communication method with a faster and secure data transfer rate. A full-scale model can be implemented for commercial purposes one this is achieved.

\section{ACKNOWLEDGMENT}

The authors wish to thank Transport Research Laboratory (TRL) for providing the opportunity to intern in Abu Dhabi. We would also like to thank the Juries of the "TRL Student Award 2016" for nominating and awarding the second place in the sustainable transportation solution category at Gulf Traffic Awards 2016.

\section{REFERENCES}

[1] H. Wang and W. He. "A reservation-based smart parking system". In Computer Communications Workshops (INFOCOM WKSHPS), 2011 IEEE Conference on, pages 690-695, April 2011.

[2] V. Srikanth, P.J. Pramod, K.P. Dileep, S. Tapas, M.U. Patil, and C.B.N. Sarat. "Design and implementation of a prototype smart parking (spark) system using wireless sensor networks". In Advanced Information Networking and Applications Workshops, 2009. WAINA '09. International Conference on, pages 401-406, May 2009.

[3] A. Gupta, V. Sharma, N.K. Ruparam, S. Jain, A. Alhammad, and M.A.K. Ripon. "Integrating pervasive computing, infostations and swarm intelligence to design intelligent context-aware parking-space location mechanism". In Advances in Computing, Communications and Informatics (ICACCI, 2014 International Conference on, pages 1381-1387, Sept 2014

[4] Chen, M. and Chang, T., 2011, June. "A parking guidance and information system based on wireless sensor network". In Information and Automation (ICIA), 2011 IEEE International Conference on (pp. 601-605). IEEE.

[5] Atif, Y., Ding, J. and Jeusfeld, M.A., 2016. "Internet of Things Approach to Cloud-based Smart Car Parking". Procedia Computer Science, 98, pp.193-198.

[6] Campbell, J., Gibbons, P.B., Nath, S., Pillai, P., Seshan, S. and Sukthankar, R., 2005, November. "Irisnet: an internet-scale architecture for multimedia sensors". In Proceedings of the 13th annual ACM international conference on Multimedia (pp. 81-88). ACM

[7] Agencies, 2013. The Telegraph U.K.. [Online] Available at: http://www.telegraph.co.uk/motoring/news/10082461/Motoristsspend-106-days-looking-for-parking-spots.html

[8] S. Mary Achkhanian, "25\% of all transportation in Dubai will be smart and driverless by 2030: Mohammad Bin Rashid", GulfNews, 2015. [Online]. Available: http://gulfnews.com/news/uae/transport/ 25-of-all-transportation-in-dubai-will-be-smart-and-driverless-by2030-mohammad-bin-rashid-1.1810896.

[9] Playground.arduino.cc. (n.d.). Arduino Playground UltrasonicSensor. [online] Available at: http://playground.arduino.cc/Main/UltrasonicSensor

[10] Arduino.cc. (n.d.). Arduino - Home. [online] Available at: https://www.arduino.cc/

[11] nRF24L01 2011. [Online]. Available:https://www.sparkfun.com/ datasheets/Components/SMD/nRF24L01Pluss_Preliminary_Product Specification_v1_0.pdf.

[12] "Raspberry Pi - Teach, Learn, and Make with Raspberry Pi", Raspberry Pi. [Online]. Available: https://www.raspberrypi.org.

[13] "Firebase | App success made simple", Firebase. [Online]. Available: https://firebase.google.com/.

[14] "Daylight Hours in Dubai, United Arab Emirates Daylength", Dubai.climatemps.com,2011. [Online]. Available: http://www.dubai.climatemps.com/sunlight.php.

[15] "11 Watt Solar Panel - Gallagher Solar Panels", Am.gallagher.com, 2009. [Online]. Available: https://am.gallagher.com/caen/products/electric-fencing/power/solar-panels/AFR11.

[16] Niculescu, A.I., Wadhwa, B. and Quek, E., 2016. Smart City Technologies: Design and Evaluation of An Intelligent Driving Assistant for Smart Parking. International Journal on Advanced Science, Engineering and Information Technology, 6(6), pp.10961102.

[17] Alawi, M.A., Alsaqour, R.A., Sundararajan, E. and Ismail, M., 2016. Prediction model for offloading in vehicular Wi-Fi network. International Journal on Advanced Science, Engineering and Information Technology, 6(6), pp.944-951. 